\title{
Chikungunya Infection: A Re-emerging Epidemic
}

\author{
Binoy J. Paul · Shajit Sadanand (D)
}

Received: May 10, 2018 / Published online: July 25, 2018

(C) The Author(s) 2018
Enhanced digital features To view enhanced digital features for this article go to https://doi.org/10.6084/ m9.figshare.6803633.

B. J. Paul

KMCT Medical College, Calicut, Kerala, India

S. Sadanand $(\bowtie)$

Government Medical College, Calicut, Kerala, India e-mail: shajitsadanand@gmail.com strategies for tackling the menace of CHIK across the globe.

Keywords: Arboviral infection; Chikungunya; Inflammatory arthritis

\section{INTRODUCTION}

Chikungunya (CHIK) is an arboviral infection caused by the chikungunya virus (CHIKV), belonging to the family Togaviridae and genus Alphavirus. The disease derives its name from "kungunyala," the Swahili word for the characteristic stooped posture of patients with the disease due to their musculoskeletal symptoms.

The first detailed description of CHIK appeared in 1952 based on an outbreak in Tanzania [1, 2]. This outbreak was followed within a span of a few years by outbreaks in South East Asia and the Indian subcontinent [3]. In India, the earliest major epidemic of CHIK was reported in 1963, in Kolkata, and a few epidemics continued thereafter to break out until 1973 [4]. After 1973 there was a period of quiescence for 32 years, with no reports of CHIK from India until 2005, when there was a largescale outbreak that began in the coastal regions of Andhra Pradesh and spread to involve approximately 16 states in India, including those in northern India as far as Delhi; a total of 1.25 million people were affected [5]. 
Manifestations of CHIK range from a mild self-limiting fever with rash and arthralgia to severe crippling acute debilitating arthritis. Musculoskeletal symptoms may persist for 3-5 years in approximately $10-60 \%$ patients [6]. The wide variation in the clinical presentation of CHIK may be the result of geographical diversity of viral strains due to the evolution of newer pathogenic strains of the virus, genetically mediated variations in the patient immune profile and/or a lack of uniform methodology in the diagnosis of CHIK and the incomplete and heterogeneous follow-up of patients in published reports [7-10].

The pan global resurgence of CHIK epidemics with their devastating economic and social impacts on developing countries in general and their overburdened health care systems in particular have been major factors in driving scientists to undertake extensive research into the evolution of CHIKV and the factors affecting the prolonged morbidity caused by this virus, with the aim to develop newer strategies to tackle this medical menace to humanity.

This review is based on previously conducted studies and does not contain any studies with human participants or animals performed by any of the authors.

\section{EPIDEMIOLOGY}

Historically, CHIK was described as early as 1779 by Dr. David Bylon who himself had contracted the disease. The earliest detailed description of CHIK in the literature was given by Robinson [1] and Lumsden [2] in 1952 regarding an outbreak in the Makonde plateau along the border of Tanzania. Outbreaks were subsequently reported from the Philippines (1954, 1956 and 1968), Thailand, Cambodia, Vietnam, India, Myanmar and Sri Lanka [3]. In India, major epidemics of CHIK were reported in 1963 in Kolkata, in 1965 in Pondicherry, Tamil Nadu, Andhra Pradesh, Madhya Pradesh and Maharashtra and again in 1973 in Maharashtra (Fig. 1). A distinctive feature of CHIKV is that it causes explosive outbreaks before apparently disappearing for several years to decades. Also, unlike dengue fever, with CHIK the dearth of asymptomatic infections leads to high attack rates which often overwhelms the health care system, as exemplified by the resurgence of CHIK in 2005 in the islands of Madagascar, Mauritius and Reunion Island [11]. In January 2006, there was a large CHIK epidemic in Reunion Island involving around $35 \%$ of the entire population followed quickly by another one in India [12]. CHIK is believed to have originated in Africa where it was maintained in 'sylvatic cycles' involving wild primates and forest dwelling mosquitoes, such as Aedes furcifer, Ae. luteocephalus, Ae. africanus or Ae. taylori [8, 9]. It was subsequently introduced into Asia where it is transmitted from human to human mainly by Ae. aegypti and, to a lesser extent, by $A e$. albopictus through an urban transmission cycle [9]. Humans act as vertebrate amplification hosts and reservoirs during epidemics, while monkeys, rodents and birds serve as reservoirs during the inter-epidemic periods [10]. Four different lineages have been identified based on genotypic and antigenic characteristics: The West African lineage, the East, Central and South African (ECSA) lineage, the Asian lineage and the Indian Ocean (IO) lineages, with the latter being monophyletic descendants of the ECSA [7, 11].

The first recorded CHIK outbreak in India was in Kolkata in 1963. This was followed by epidemics in Tamil Nadu, Andhra Pradesh and Maharashtra in 1964-1965 and in Barsi in 1973 [13] and then by a period of quiescence when CHIKV seemed to have disappeared from India. After a gap of 32 years, the virus re-emerged in 2006 and wreaked havoc in India by causing an epidemic that involved more than a million people across 16 states, ranging from the southern states of Kerala, Tamil Nadu, Andhra Pradesh, Karnataka, Maharashtra, Madhya Pradesh and Gujarat to more northern regions such as Delhi. A mutation found in the E1 protein of CHIKV (A226V) removes the requirement of this virus for cholesterol in the cell membrane (cholesterol dependence) for virus fusion, resulting in better viral uptake, replication and transmission by Ae. albopictus, which has a wider geographical distribution than does $A e$. aegypti [12-14]. Additional adaptive mutations have also been identified in CHIKV that involve 


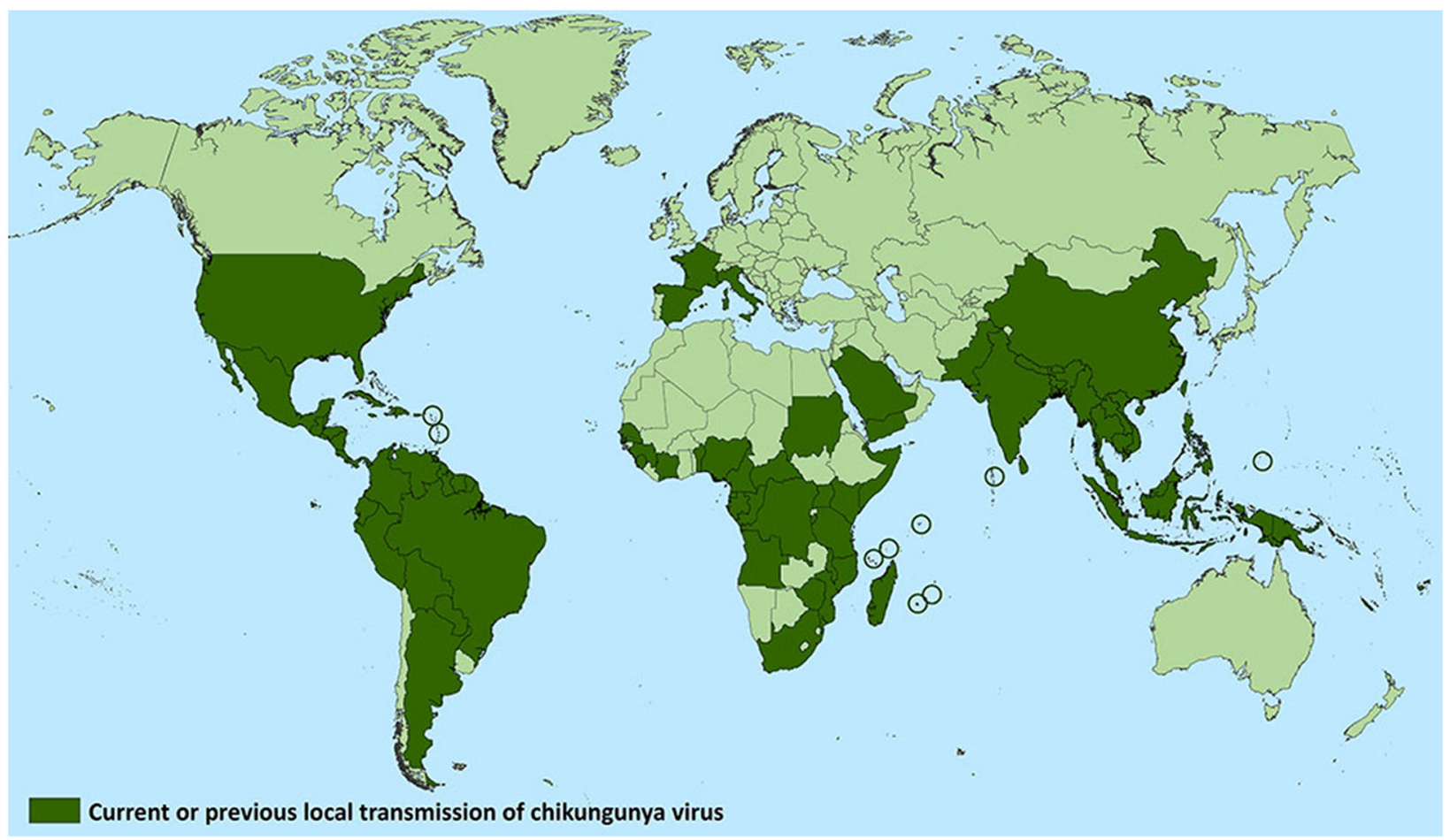

Fig. 1 Countries which have reported the occurrence of Chikungunya (both recently and historically). Source U.S. Centers for Disease Control and Prevention website. https://www.cdc.gov/chikungunya/geo/index.html. Accessed 1 July 2018

E2 and E3 substitutions; these enhance the initial infection of the Ae. albopictus midgut by CHIKV leading to greater vector competence [47].

After 2006, suspected cases of CHIK have reported from many states across India. Around 28,000 suspected CHIK cases were reported in 2015. In 2016 and 2017 there was a large upsurge of CHIK cases, with 64,057 cases in 2016 and 62,268 cases in 2017 reported from different parts of the country [15].

\section{ETIOLOGY}

\section{Chikungunya virus}

Chikungunya virus was first isolated in Tanzania by Ross and colleagues [48]. It is an arbovirus that belongs to the genus Alphavirus in the Togaviridae family. The virus has a diameter of 60-70 nm, a positive-sense, single-stranded
RNA genome with 11,438 nucleotides and a phospholipid envelope with hemagglutinin protein spikes $[16,17]$.

\section{Host and the Vector}

Two different transmission cycles exist for the CHIKV, namely the sylvatic cycle seen mainly in Africa and the urban cycle that was initially seen in Asia but is also now found in Africa. CHIKV is maintained in nature by the sylvatic cycle, with monkeys, rodents, baboons and birds serving as reservoir hosts. Human beings replace these wild animals as reservoirs during periods of epidemics [10] The most effective vector for human transmission is Ae. aegypti [18], while Ae. furcifor-taylori is the predominant species for transmission in animals [18]. Although Ae. albopictus is readily infected by CHIKV, its transmissibility is low [19]. However a mutation found in the E1 protein of CHIKV 
(A226V) has enabled this virus to eliminate its cholesterol dependence, thereby achieving better viral uptake, replication and transmission by Ae.. albopictus, which has a wider geographical distribution than Ae. aegypti. This led to an explosive outbreak of CHIK in the Indian Subcontinent post 2006 [20]. The vector competency of Ae. albopictus has also been enhanced by adaptive mutations in CHIKV involving E2 and E3 substitutions which enhance the initial infection of the Ae. albopictus midgut by CHIKV [47].

The persistence of the CHIKV virus in urban cycle is thought to be the result of the continuous introduction of CHIKV to immunologically naive populations. Vertical transmission from an infected mother to her infant has been described during the epidemic in the Reunion Islands. The greatest risk for this transmission appears to be during birth [21]. An unusual characteristic of CHIK infection is its periodicity, with intervals of quiescence, sometimes extending up to decades. Possible explanations for this phenomenon seems to be variations in herd immunity, genomic alterations in the virus and ecological factors associated with urbanization, migration and deforestation. Outbreaks are most likely to occur in the postmonsoon period when the vector density is very high, a factor which accentuates the transmission.

\section{PATHOGENESIS}

After subcutaneous inoculation through the bite of an infected mosquito, CHIKV disseminates through the lymph nodes and microvasculature. The entry of the virus into the lymph nodes is facilitated by the Langerhans cells. The primary sites of viral replication are the liver, spleen and lymph nodes, thus allowing an efficient viraemia. The acute phase of the illness involves viral replication followed by an efficient inflammatory response in the target tissues utilizing macrophages (the main component) along with neutrophils, lymphocytes and NK cells. The resulting rise in pro-in- flammatory cytokines and chemokines at the infection site, local tissues and systemic circulation is responsible for the systemic manifestations, such as fever, myalgia and arthralgia [5]. The secretion of metalloproteinase (MMP) in the joint tissues also contributes to the articular damage. Persistence of the virus or its products in the target cells and the resultant accumulation of inflammatory mediators, such as interleukin-6 (IL-6) and granulocyte-macrophage colony-stimulating factor (GM-CSF), contribute to persistent symptoms and chronic arthritis.

CHIKV, similar to other arboviruses, are potent inducers of interferon (INF) which in turn inhibits viral replication. Macrophages, which are a major source of INFs, are readily infected by the CHIKV and they play a pivotal role in several cellular responses, as shown in experimental studies, and in the responses of both the Th1- and Th2-type cytokines to CHIKV $[22,23]$. It is hypothesized that type 1 IFNs mediate antiviral response while type II IFNs $($ IFN- $\gamma)$, which are produced in the early stages of CHIKV infection, further promote the transition from innate to adaptive immunity [24]. It has also been shown that CD4 + T cells induced by CHIKV infection are the major producers of IFN- $\gamma$ and that Th1 cells are likely responsible for a skewed production of immunoglobulin (Ig) G2 antibodies by B cells in response to IFN- $\gamma$ [25]. IL-13, which is an important immunomodulatory cytokine, also induces B-cell proliferation and IgE switching and has been speculated to be associated with persistent arthritis in CHIKV [26].

\section{CLINICAL FEATURES}

Clinical manifestations of CHIK are variable, ranging from asymptomatic infection in a minority of patients to severe crippling debilitating illness. Manifestations are often of acute onset, developing after an incubation period of 4-7 (range 2-12) days. The disease is self-limiting in the majority of patients, with the symptoms disappearing within 7-10 days. Viraemia 
may persist for 5 days from the onset of symptoms [27].

A clinical triad of 'fever, rashes and arthralgia' is suggestive of CHIK fever. Fever is typically abrupt in onset and usually lasts for 4-5 days. It may be either high grade or low grade and can be associated with chills and rigor. In a study by the authors involving 150 patients with CHIK, fever subsided within 2 days in $43.8 \%$ of patients and in 3-5 days in $49.2 \%$ [28]. In around $30 \%$ of patients, fever may return back after subsiding for 1-2 days, giving a classic saddle back pattern [29].

Polyarthralgia begins 2-5 days after the onset of fever and is mostly symmetrical, involving predominantly the distal joints including wrists, ankles and small joints of hands [7]. Arthritis may sometimes involve the large joints, such as the knee and shoulder. CHIK arthritis has also a predilection to affect both early and more severely those joints previously damaged due to trauma or degeneration [27]. The joint pain is particularly devastating in terms of severity, almost immobilizing the patient and at times even preventing sleep during the first few days of the illness. The severe pain leads to the characteristic stooped posture from which the disease derives its name. Joints are extremely tender to touch in many patients [30]. The arthritis persists for varying periods of time. In the pervious study conducted by the authors, the arthritis resolved within 1 week in $25 \%$ of patients and within 1 month in $60 \%$ [28]. The most common joints affected in that patient population were the ankle (98\%) and small joints of hands (93\%) [28]. Periarticular soft tissue swelling with or without tenderness is a common feature of CHIK arthritis and close to $90 \%$ patients in the authors' study had this manifestation; spine and sacroiliac joints were rarely involved although $21 \%$ patients had history of low back ache at disease onset [28].

Arthritis associated with CHIK is generally a self-limiting disease. However, in a small percentage of patients, long-term sequelae can be seen, ranging from episodic stiffness to persistent stiffness and restriction of movements [27]. In the authors' previous study, the most common joints affected with chronic arthritis were the ankle (63\%), followed by the knee (55\%) and small joints of the hands (50\%) [28]. Tenosynovitis was present in 5\% patients with chronic arthritis. Apart from chronic arthritis, CHIK can evolve or precipitate rheumatoid arthritis, as was noted by the authors in $5 \%$ of patients on follow-up [28].

Dermatological manifestations are a prominent feature of CHIK fever. Transient maculopapular rash may be seen in approximately $50 \%$ patients [27]. Nasal blotchy erythema, centrofacial hyperpigmentation, lichenoid eruptions, hyperpigmentation in photodistributed areas and exfoliative dermatitis are other dermatological manifestations observed [27]. In the authors' previous study, rash was present in $68 \%$ patients, which was erythematous $(45 \%)$, macular $(42 \%)$ and papular in pattern (20\%) [28]. An unusual centrofacial rash was observed in $21 \%$ patients, which began as an erythematous rash at the tip of the nose and malar eminences and later became hyperpigmented, resembling the malar rash of systemic lupus erythematosus. Desquamation of ear lobules, palms and soles was observed in $26 \%$ of patients, and pruritus with or without rash was seen in 29\% [28]. Mucosal lesions in the form of aphthous ulcers, lip ulcers and glossitis were also observed [28].

Other systemic manifestations of CHIK include headache, asthenia, myalgia, vomiting and lymphadenopathy [28, 31]. Although alphaviruses are notorious in causing neurological manifestations, neurological complications are not common with CHIK infection.

Vertical transmission from infected mothers to the fetus may occur, especially if the mother is viraemic during the perinatal period [32]. Manifestations are usually mild in these cases, with fever, irritability and bullous dermatitis $[7,27]$.

\section{DIAGNOSIS}

\section{Laboratory Diagnosis}

As there is considerable overlap between the clinical manifestations of CHIK and those of other viral fevers, such as dengue, laboratory 
diagnosis is crucial in differentiating the doubtful cases to initiate public health measures and to provide clinical clues (Table 1).

Routine blood tests reveal lymphopenia consistent with that of a viral fever. However, thrombocytopenia is not a prominent finding and its presence would help to differentiate CHIK from dengue fever [27]. Also, the erythrocyte sedimentation rate and acute phase reactants, such as C-reactive protein, would show moderate to marked elevation in CHIK, unlike dengue and other viral fevers. Mild elevations in liver enzymes, such as like serum glutamic oxaloacetic transaminase and serum glutamic pyruvic transaminase, are seen in CHIK infections. Similar patterns were observed by the authors in their study on 150 cases of CHIK in a tertiary care hospital [28].

Definitive laboratory diagnosis can be accomplished through three main laboratory tests: virus isolation, serological test and PCR analysis [27]. Specimens used are blood or serum, or cerebro-spinal fluid in the case of meningoencephalitis.

\section{Virus Isolation}

Virus isolation is the gold standard test for diagnosis, but the result is only available after several days, and the virus can only be isolated in specialized biosafety level III laboratories due to the risks of transmission involved. The technique involves exposing specific cell lines or infant mice to samples from whole blood and

Table 1 Clinical clues which help to differentiate Chikungunya and Dengue fever

\begin{tabular}{|c|c|c|}
\hline Clinical feature & Chikungunya & Dengue fever \\
\hline High fever & Present & Present \\
\hline Myalgia & Less common & More common \\
\hline Arthralgia & More common & Less common \\
\hline Retro-orbital pain & Less common & More common \\
\hline Characteristic pattern of rash & $\begin{array}{l}\text { Centrofacial } \\
\text { hyperpigmentation }\end{array}$ & Erythematous maculo-papular "White Islands in Red Sea" \\
\hline Desquamation & More common & Unlikely \\
\hline Bleeding manifestation & Uncommon & More common \\
\hline Hypotension/shock & Uncommon & More common \\
\hline Leukopenia & Less common & More common \\
\hline Neutropenia & Less common & More common \\
\hline Lymphopenia & More common & Less common \\
\hline Thrombocytopenia & Less common & More common \\
\hline Raised ESR/CRP & More common & Less common \\
\hline Liver enzymes SGOT/SGPT & $\begin{array}{l}\text { Mild elevation (100-200 IU/ } \\
\text { L) }\end{array}$ & Moderate elevation $(100-500 \mathrm{IU} / \mathrm{L})$ \\
\hline Serology & IgM CHIK & IgM Dengue \\
\hline PCR & CHIK RT-PCR & Dengue RT-PCR \\
\hline
\end{tabular}

ESR Erythrocyte sedimentation rate, CRP C-reactive protein, $S G O T$ serum glutamic oxaloacetic transaminase, $S G P T$ serum glutamic pyruvic transaminase, $I g$ immunoglobulin, CHIK Chikungunya, $R T$ reverse transcriptase 
identifying CHIKV-specific responses. Positive results depend on a multitude of factors, such as proper sample collection and cold chain maintenance during transport to these laboratories [27].

\section{Serological Test}

Sero-diagnosis relies on the ability to demonstrate a fourfold increase in CHIK IgG titre between the acute and convalescent phase sera or to detect IgM anti CHIKV antibodies in acute phase sera, in the absence of concurrent circulation of other agents producing a similar disease syndrome (in which case CHIKV infection would have occurred up to months earlier and another infection may be the current infection). IgM anti-CHIKV antibodies are elevated in the blood of infected patients as early as at 5 days of infection and they remain elevated for 3-6 months [27, 33]. Anti-CHIKV IgG is detectable only after 2 weeks of infection and remains elevated for 6 months.

Capture enzyme-linked immunosorbent assays (ELISA) and immune-chromatographic tests are two common techniques for estimating CHIKV titres in blood [33]. The ELISA is generally quite specific for CHIKV, but plaque-reduction neutralization is the gold standard for serology.

\section{Nucleic Acid Detection-PCR}

Early diagnosis of CHIKV is possible by nucleic acid detection techniques, such as reverse transcription (RT) PCR and real-time loop-mediated isothermal amplification (RT-LAMP) methods [7, 34, 35].The advantages of RT-LAMP is that it does not require a PCR instrument as the assay is carried out in a water bath [35].

The use of cytokines and other biomarkers to aid in the diagnosis of CHIK infection is hampered by the lack of validation and heterogeneity in the cytokine response across various populations [7].

\section{Diagnostic Criteria for CHIK Infection}

A probable or suspected case is defined as a patient who meets the clinical criteria only. A confirmed (definitive) case is defined as a patient who meets both the clinical and laboratory criteria [27].

\section{Clinical criteria}

The clinical criteria for diagnosing CHIK infection include acute onset of fever and severe arthralgia/arthritis with or without skin rash and residence in an epidemic area or having been in and subsequently having left an epidemic area 15 days prior to onset of symptoms.

\section{Laboratory criteria}

The laboratory criteria include at least one of the following results from tests performed in the acute phase of illness.

1. Virus isolation/presence of viral RNA by RTPCR.

2. Presence of virus specific IgM antibodies in single serum sample collected in the acute or convalescent stage.

3. Fourfold increase in IgG values in samples collected at least 3 weeks apart.

\section{MANAGEMENT}

Management of CHIK is usually supportive with adequate rest, hydration, antipyretics and analgesics (nonsteroidal anti-inflammatory drugs [NSAIDs]). To date, no vaccines have been approved for the prevention of CHIKV infection [7].

Chloroquine phosphate (CQ) has been used effectively to manage CHIKV arthritis [36]. In a previous study, the authors used hydroxychloroquine (HCQ) to treat patients with chronic CHIK arthritis, with $79 \%$ of patients showing a good response [28]. A 24-week, twoarm parallel efficacy trial comparing CQ and meloxicam did not show any significant difference in efficacy between the two drugs [37]. A very recent randomized controlled trial compared combinations of disease-modifying antirheumatic drugs (DMARDs), namely, methotrexate, sulfasalazine, and HCQ with HCQ monotherapy, and found better efficacy with DMARDs [38]. Ganu et al. found good response with a combination of sulfasalazine and methotrexate compared to sulfasalazine alone in patients with chronic persistent CHIK 
arthritis not responding to NSAIDs and HCQ for 3 months [39].

A recent article on CHIK mentioned the use of a low-dose steroid regime $(<0.5 \mathrm{mg} / \mathrm{kg}$ deflazocort) with rapid tapering over 6 weeks for the management of acute CHIK infection. The same was used 1 week after NSAID use after the period of viraemia was over [7]. In their previous study, the authors had used low-dose steroids in $9 \%$ patients in whom the symptoms were severe and not controlled on NSAIDs and/ or HCQ [28]. The French Infectious Disease Society recommends the use of NSAIDs and low-dose steroids as initial options for postacute and chronic CHIK infections with the option of methotrexate in patients not responding to initial therapy [40].

The role of DMARDs in acute CHIK arthritis is uncertain. HCQ takes weeks to produce its effect, by which time the arthritis has resolved in the majority of patients. Also, the majority of DMARDs have a COX-2 inhibitory action and may themselves induce NSAID-like effects if used early in the disease course before the arthritis self-remits [7].

In one study, ribavarin $200 \mathrm{mg}$ twice daily for 1 week was tried on ten patients with chronic CHIK compared to placebo, with some effect [41]. Other promising molecules reported to have some efficacy in CHIK are bindarit [42], an inhibitor of monocyte chemoattractant protein-1 (MCP-1), inhibitors targeting the mannose-binding lectin (MBL) pathway of the complement system [43] and beclin, which is an autophagy-inducing peptide [44]. Other potential drugs with anti-CHIK action are niclosamide, nitazoxanide and suramin, all of which inhibit virus entry, fusion, binding and cell-tocell transmission $[45,46]$.

\section{CHALLENGES FOR FUTURE}

Chikungunya infection is an important threat to mankind in view of the pronounced, longlasting musculoskeletal morbidity and loss of productivity caused by it. A greater understanding of the pathogenesis and recognition of novel markers implicated in the viral persistence and progression of the disease into disabling arthritis is a realistic goal. Newer research avenues with the development of preventive strategies would go a long way to tackle this menace.

\section{ACKNOWLEDGEMENTS}

Funding. No funding or sponsorship was received for this study or publication of this article.

Authorship. All named authors meet the International Committee of Medical Journal Editors (ICMJE) criteria for authorship for this article, take responsibility for the integrity of the work as a whole, and have given their approval for this version to be published.

Disclosures. Binoy J. Paul and Shajit Sadanand have nothing to disclose.

Compliance with Ethics Guidelines. This article is based on previously conducted studies and does not contain any studies with human participants or animals performed by any of the authors.

Open Access. This article is distributed under the terms of the Creative Commons Attribution-NonCommercial 4.0 International License (http://creativecommons.org/licenses/ by-nc/4.0/), which permits any noncommercial use, distribution, and reproduction in any medium, provided you give appropriate credit to the original author(s) and the source, provide a link to the Creative Commons license, and indicate if changes were made.

\section{REFERENCES}

1. Robinson MC. An epidemic of virus disease in Southern Province, Tanganyika Territory, in 1952-53; I. Clinical features. Trans R Soc Trop Med Hyg. 1955;49:28-32.

2. Lumsden WHR. An epidemic of virus disease in Southern Province, Tanganyika Territory, in 
1952-53; II. General description and epidemiology. Trans R Soc Trop Med Hyg. 1955;49:33-57.

3. World Health Organization. Chikungunya. Geneva: World Health Organization; 2013. http://www. who.int/mediacentre/factsheets/fs32.

4. Sudeep AB, Parashar D. Chikungunya: an overview. J Biosci. 2008;33:443-9.

5. Lahariya C, Pradhan SK. Emergence of chikungunya infection in India after 32 years: a review. J Vect Borne Dis. 2006;43:151-60.

6. Schilte C, Staikowsky F, Couderc T, et al. Chikungunya virus-associated long-term arthralgia: a 36 month prospective longitudinal study. PLoS Negl Trop Dis. 2013;7:e2137. https://doi.org/10. 1371/journal.pntd.0002137.

7. Mathew A, Ganapati A, Kabeerdoss J, et al. Chikungunya infection: a global public health menace. Curr Allergy Asthma Rep. 2017;17(2):13. https://doi.org/10.1007/s11882-017-0680-7.

8. Brooks GF, Butel JS, Morse SA (eds). Human arboviral infections. In: Jawetz, Melnick and Adelberg's medical microbiology. 23rd edn. Singapore: McGraw Hill; 2004: 514-24.

9. Jupp PG, McIntosh BM. Chikungunya disease. In: Monath TP, editor. The arboviruses: epidemiology and ecology. Boca Raton: CRC Press; 1988::137-57.

10. Caglioti C, Lalle E, Castilletti C, Carletti F, Capobianchi MR, Bordi L. Chikungunya virus infection: an overview. New Microbiol. 2013;36:21127.

11. Cavrini F, Gaibani P, Pierro AM, Rossini G, Landini MP, Sambri V. Chikungunya: an emerging and spreading arthropod-borne viral disease. J Infect Dev Ctries. 2009;3:744-52.

12. Yergolkar PN, Tandale BV, Arankalle VA, Sathe PS, Sudeep AB, Gandhe SS, et al. Chikungunya outbreaks caused by African genotype, India. Emerg Infect Dis. 2006;12:1580-3.

13. Parashar D, Patil D. Chikungunya: a disease reemerged in India after 32 years. A review in Diamond jubilee publication of NIV Commemorative compendium. In: Arankalle VA, Cecilia D, editors. NIV Golden to diamond jubilee: the glorious decade. 2012. p. 221-42.

14. Cherian SS, Walimbe AM, Jadhav SM et al. Evolutionary rates and timescale comparison of Chikungunya viruses inferred from the whole genome/E1 gene with special reference to the 2005-07 outbreak in the Indian subcontinent. Infect Genet Evol. 2009;9:16-23.
15. National Vector Borne Disease Control Programme. Chikungunya fever. 2017. http://nvbdcp.gov.in/ chikun-status.html. Accessed 30 Dec 2017.

16. Khan AM, Morita K, Parquet MC, Hasibi F, Edward GM, Igarshi A. Mathange. Complete nucleotide sequence of Chikungunya virus and evidence for an internal polyadenylation site. J Gen Virol. 2002;83:3075-84.

17. Barret ADT, Weaver SC. Arboviruses: alphaviruses, flaviviruses and bunyaviruses. Medical Microbiology. Churchill Livingstone 2002; 484-501.

18. Mourya DT, Yadav P. Vector biology of dengue and chikungunya. Indian J Med Res. 2006;124:475-80.

19. McIntosh BM, Jupp PG. Attempts to transmit chikungunya virus with six species of mosquito. J Med Ent. 1970;7:615-8.

20. Pradeep K, Joseph R, Kamaraj T, Jambulingam P. A226V mutation in virus during 2007 chikungunya outbreak in Kerala, India. J Gen Virol. 2008;89:1945-8.

21. Gérardin P, Barau G, Michault A, et al. Multidisciplinary prospective study of mother-to-child chikungunya virus infections on the island of La Réunion. PLoS Med. 2008;5:e60.

22. Couderc T, Chrétien F, Schilte C, et al. (2008) Mouse model for chikungunya: young age and inefficient type-1 interferon signaling are risk factors for severe disease. PLoS Pathog 4(2)e29:0001-0012.

23. Ziegler SA, Lu L, Travassos RA, Xiao SY, Tesh RB. An animal model for studying pathogenesis of Chikungunya virus infection. Am J Trop Med. 2008;79(1):133-9.

24. Schilte C, Couderc T, Chretien F, et al. Type I IFN controls chikungunya virus via its action on nonhematopoietic cells. J Exp Med. 2010;207(2):429-42.

25. Barr TA, Brown S, Mastroeni P, Gray D. B cell intrinsic MyD88 signals drive IFN-gamma production from $\mathrm{T}$ cells and control switching to IgG2. J Immunol. 2009;183:1005-12.

26. Venugopalan A, Ghorpade RP, Chopra A. Cytokines in acute Chikungunya. PLoS One. 2014;24:e111305.

27. National Vector Borne Disease Control Programme. Chikungunya fever-national guidelines. nvbdcp.gov.in/Doc/National-Guidelines-Clinical-Management-Chikungunya-2016.pdf. 
28. Paul B, Pannarkady G, Moni S, Thachil E. Clinical profile and long-term sequelae of Chikungunya fever. Indian J Rheumatol. 2011;6(1):12-9.

29. National Institute of Communicable Disease, New Delhi. Chikungunya Fever. CD Alert. 2006;10(2):6-8.

30. Paquet C, Quatresous I, Solet JL, et al. Chikungunya outbreak in reunion: epidemiology and surveillance. Euro Surveill. 2006;11:2.

31. Cecilia D. Current status of dengue and chikungunya in India. WHO South-East Asia J Public Health. 2014;3(1):22.

32. Sebastian M, Lodha R, Kabra S. Chikungunya infection in children. Indian $\mathrm{J}$ Pediatr. 2009;76(2):185-9.

33. Prince HE, Seaton BL, Matud JL, Batterman HJ. Chikungunya virus RNA and antibody testing at a national reference laboratory since the emergence of Chikungunya virus in the Americas. Clin Vaccine Immunol. 2015;22:291-7.

34. Patel P, Abd El Wahed A, Faye O, et al. A field-deployable reverse transcription recombinase polymerase amplification assay for rapid detection of the Chikungunya virus. PLoS Negl Trop Dis. 2016;10:e0004953.

35. Parida MM, Santhosh SR, Dash PK, et al. Rapid and real-time detection of Chikungunya virus by reverse transcription loopmediated isothermal amplification assay. J Clin Microbiol. 2007;45:351-7.

36. Brighton SW. Chloroquine phosphate treatment of chronic Chikungunya arthritis. An open pilot study. S Afr Med J. 1984;66:217-8.

37. Chopra A, Saluja M, Venugopalan A. Effectiveness of chloroquine and inflammatory cytokine response in patients with early persistent musculoskeletal pain and arthritis following chikungunya virus infection. Arthritis Rheumatol. 2014;66:319-26.

38. Ravindran V, Alias G. Efficacy of combination DMARD therapy vs hydroxychloroquine monotherapy in chronic persistent chikungunya arthritis: a 24-week randomized controlled open label study. Clin Rheumatol 2017;36(6):1335-40.

39. Ganu MA, Ganu AS. Post-chikungunya chronic arthritis-our experience with DMARDs over two year follow up. J Assoc Phys India. 2011;59:83-6.

40. Simon F, Javelle E, Cabie A, et al. French guidelines for management of chikungunya (acute and persistent presentations), November 2014. Med Mal Infect. 2015;45:243-63.

41. Ravichandran R, Manian M. Ribavirin therapy for Chikungunya arthritis. J Infect Dev Ctries. 2008;2:140-2.

42. Rulli NE, Rolph MS, Srikiatkhachorn A, Anantapreecha S, Guglielmotti A, Mahalingam S. Protection from arthritis and myositis in a mouse model of acute chikungunya virus disease by bindarit, an inhibitor of monocyte chemotactic protein-1 synthesis. J Infect Dis. 2011;204:1026-30.

43. Gunn BM, Morrison TE, Whitmore AC, et al. Mannose binding lectin is required for alphavirusinduced arthritis/myositis. PLoS Pathog. 2012;8:e1002586.

44. Shoji-Kawata S, Sumpter R, Leveno M, et al. Identification of a candidate therapeutic autophagy-inducing peptide. Nature. 2013;494:201-6.

45. Wang Y, Lu J, Lin C, et al. Antiviral activities of niclosamide and nitazoxanide against chikungunya virus entry and transmission. Antiviral Res. 2016;135:81-90.

46. Ho Y, Wang Y, Lu J, et al. Suramin inhibits chikungunya virus entry and transmission. PLoS One. 2015;10(7):e0133511.

47. Lounibos LP, Kramer LD. Invasiveness of Aedes aegypti and Aedes albopictus and vectorial capacity for Chikungunya virus. J Infect Dis. 2016;214[Suppl 5]:S453-8. https://doi.org/10.1093/infdis/jiw285.

48. Ross RW (1956) The Newala epidemic. III. The virus: isolation, pathogenic properties and relationship to the epidemic. J Hyg (Lond) 54(2):177-91. 